\title{
A Multistage Approach for Quality- and Efficiency-Related Tailoring of Modelling and Simulation Processes
}

\author{
Zhongshi Wang, Axel Lehmann, Alexandros Karagkasidis, Univ. der Bundeswehr München, Germany
}

\{zhongshi.wang, axel.lehmann, alexandros.karagkasidis\}@unibw.de

SNE Simulation Notes Europe SNE 19(2), 2009, 12-20, doi: 10.11128/sne.19.tn.09933

For reasons of increasing productivity, efficiency, and decreasing time and cost for complex system innovations, modelling and simulation (M\&S) becomes a standard "tool" for a huge variety of applications. The demand for controlling and demonstrating the quality of a model and its applications by introducing appropriate quality measures, techniques, and tools is obvious. Consequently, a variety of structured and even standardized processes for development of modelling and simulation (M\&S) applications as well as for verification and validation $(\mathrm{V} \& \mathrm{~V})$ has been proposed. All of them require, however, due to different characteristics of organisation structures and project environments, some kind of adaptation or tailoring prior to application. This paper describes a multistage approach for tailoring of an M\&S project in compliance with the principles of the V-Modell XT, which is considered as the German standard IT development process obligatory for federal engineering projects. Along with the integration of the M\&S-specific components into the VModell, this tailoring approach enables the project-specific selection of essential products, documents and activities for developing M\&S applications and conducting their V\&V according to specified cost, time and application constraints.

\section{Introduction}

Enabled by rapid advances of computer and network technologies, the development and application of increasingly complex simulation models and applications $(\mathrm{M} \& \mathrm{~S})$ in various domains is inevitable. Accompanied by increasingly powerful visualisation and simulation infrastructures and tools, quality assurance, especially correctness and validity of models and of simulation results becomes an urgent requirement.

As a consequence, a large number of well-structured and even standardized processes [1, 2, 3, 4, 5, 6] for development of $M \& S$ applications and also for their verification and validation $(\mathrm{V} \& \mathrm{~V})$ have been introduced. However, since there exist no two organisations with the same structural properties, and furthermore, every M\&S project differs in terms of objectives, scale, scope, technical challenges etc., none of these processes is well suited for all possible circumstances. Therefore, the potential of an M\&S process to be adapted to various application domains and different project environments is a crucial issue for its acceptance in practical application [7, 8].

Instead of proposing new standard processes, this work investigates the opportunity to use the V-Modell XT $[9,10,11,12]$ for conducting an M\&S project, and introduces a multistage approach to adaptation of model development and $\mathrm{V} \& \mathrm{~V}$ to the actual project context in accordance with the principles of the $\mathrm{V}$ Modell. As the official German standard development process for IT systems, the V-Modell describes detailed requirements and guidelines necessary for system development throughout the entire life cycle, integrating various essential management processes, such as quality assurance, project management, and configuration management. It is based on a modular structure, and can be flexibly extended. A considerable amount of benefits can be directly achieved by using the V-Modell for M\&S development, since:

- the V-Modell covers all relevant aspects for software and hardware development;

- the V-Modell XT can be flexibly adapted to project-specific constraints due to its advanced tailoring concept;

- documentation, templates, training material, and open source tools are publicly available;

- supporting tools developed for the V-Modell could be also applied to model development;

- comprehensive experiences collected from VModell applications in practice are also meaningful for model development;

- the V-Modell XT obtains growing (national as well as international) acceptance.

As already discussed in [13, 14], since some essential elements for conducting a simulation study are not available in the V-Modell XT, the M\&S-specific components have to be additionally defined and integrated. Therefore, the scope of adaptation addressed 
in the context of this paper is twofold: (1) enabling the V-Modell XT to conduct an M\&S project; (2) tailoring of an M\&S project with respect to specified time, cost and application constraints.

The remainder of this paper begins with a brief introduction to the V-Modell XT. Section 2 points out the required $M \& S$-specific aspects to be integrated in the V-Modell. In Section 3, the adaptations of the VModell for conducting an M\&S project and a multistage tailoring approach are introduced. Section 4 presents an application example of the tailoring process. Finally, Section 5 concludes the work.

\section{Overview of the V-Modell XT}

The V-Modell XT defines detailed requirements and guidelines necessary for developing a successful software and hardware system throughout the entire project life cycle from the project planning up to the acceptance of end products. The following description provides a general outline of the basic concepts included in the V-Modell [9, 12], particularly the concepts of Project Types, Process Modules, Project Execution Strategies and Tailoring.

\subsection{Project Types and Project Type Variants}

The success of a system development depends on whether the user requirements are fulfilled to the full extent. Therefore, clients should be involved in the entire project life cycle and work closely with other project participants. According to the particular perspectives of clients and contractors on the development process, the V-Modell XT distinguishes different project types, each of which defines a set of specific tasks from the viewpoint of a client (acquirer) or a contractor (supplier), concerning the different project execution. To describe project characteristics in more detail, different project type variants are defined for each project type. A project type variant specifies the basic requirements for a possible project execution regarding contents and time, and thus, determines an ordered development life cycle.

\subsection{Process Modules}

The essential structure of the V-Modell is represented by various process modules. Each process module focuses on a particular task to be accomplished within the scope of a V-Modell project, and defines a set of products, activities and roles required to complete the intended task in the context of a certain process area, such as project management, software development, quality assurance, etc.

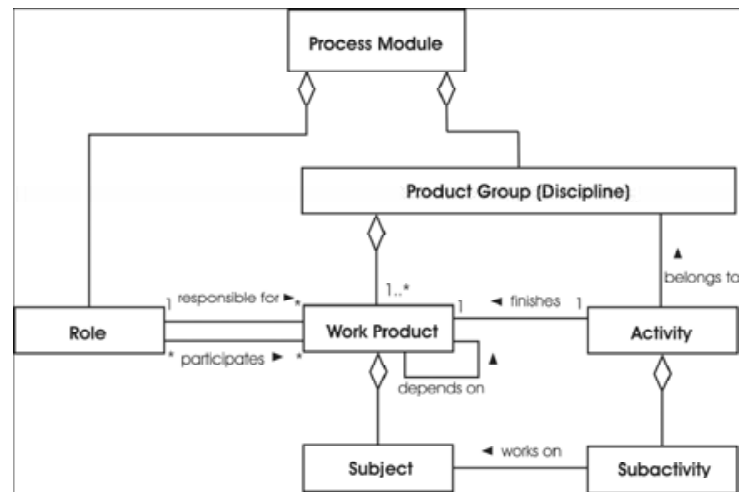

Figure 1. Structure of a process module.

Figure 1 shows the structure of a process module defined in the V-Modell. Products stand in the central point and represent the main project results. Each work product being developed is completed exactly by one activity. To define the responsibility of product creation, a well-structured role concept is introduced. During a project execution, a person or an organisational unit can be assigned to a role, according to the competency. Several roles may contribute to a product's creation concurrently, but only one of them carries the responsibility. A product can be subdivided further into several subjects. In addition, products can be also integrated into a product group (or a discipline) with regard to contents, and the associated activities belong to the same discipline.

The concept of process modules is a typical component-based approach [10]. Each process module contained in the V-Modell is an independent unit, and can be changed and extended for different project situations. The four process modules: Project Management, Quality Assurance, Configuration Management, and Problem and Change Management specified in the V-Modell XT, are mandatory to be used for each software development project, and therefore, are also designated as the core process modules or the VModell Core.

\subsection{Project Execution Strategies and Decision Gates}

Since a process module does not contain any information about the order of preparing certain products, project execution strategies are introduced to specify the possible sequence of product development. With respect to time aspect, each project execution strategy represents an ordered development life cycle for a certain project type, and defines in detail the different project progress stages to be achieved, which are designated as decision gates. A decision gate is com- 
parable with a milestone in a project, and indicates which products have to be finished in which order [10]. To achieve the decision whether the defined products have been completed correctly and accurately, diverse verification and validation (V\&V) methods must be applied to evaluate the quality of products. The relationship between project types, project type variants, process modules, and project execution strategies is illustrated in Figure 2.

\subsection{Tailoring}

According to specified cost, time and application constraints, the V-Modell XT can be adapted to different project constellations. This project-specific adaptation is called tailoring. The tailoring process of the V-Modell begins with the selection of a project type. Since a set of process modules is predefined for each project type, this step determines mandatory process modules preliminarily. After that, a possible project type variant of the selected project type is to be determined, by means of which not only the project execution strategy but also further process modules can be selected.

Moreover, different project characteristics describing the project in more detail are assigned to the selected project type and the associated project type variant. During the Tailoring process, one value that has to be selected from a number of possible values must be determined for each project characteristic. This step could add additional process modules and modify the project execution strategy. As a result, besides the VModell Core only relevant process modules and a suitable project execution strategy with well-defined decision gates are determined exactly in consideration of the actual project conditions. The work process for further tailoring of products and activities within a process module is, however, not defined in the standard V-Modell XT.

\section{Modelling and Simulation in the Context of the V-Modell XT}

Modelling and Simulation (M\&S) requires a specific development process, which can not be completely specified by the V-Modell XT [13, 14]. This section investigates the essential features of an M\&S project, and points out the M\&S-specific elements which have to be additionally defined and integrated in the V-Modell for conducting a simulation study. The resultant new variant of the V-Modell is referred to as the V-Modell XT-M\&S.

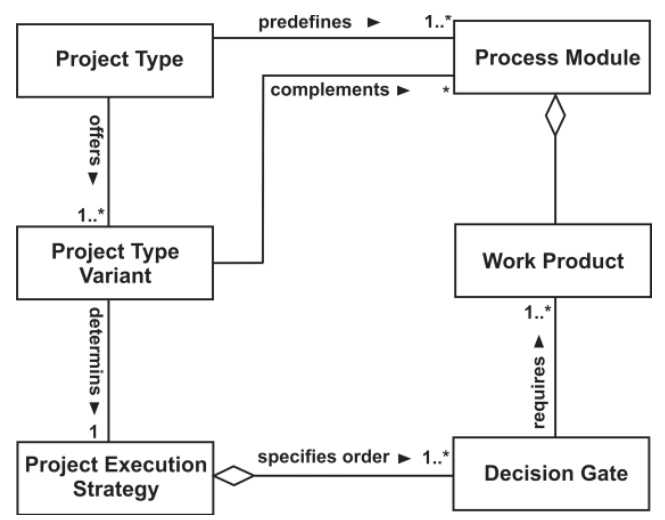

Figure 2. Relationship between the basic concepts.

\subsection{Adding the M\&S-specific Aspects: towards the V-Modell XT-M\&S}

Commonly, regardless of how a concrete modelling process looks like, a simulation model is developed through the progress stages of Model Initialisation, Model Design, Model Realisation and Model Application in the course of any M\&S project. For each progress stage, one or more (intermediate) work products are to be prepared, and the quality of them is also to be estimated as part of the model development. For example as shown in Figure 3, the progress stage Model Initialisation includes the work product Sponsor Needs (SN), in Model Design the products Structured Problem Description (SPD), Conceptual Model (CM) and Formal Model (FM) are defined, Executable Model (EM) is prepared in Model Reali-

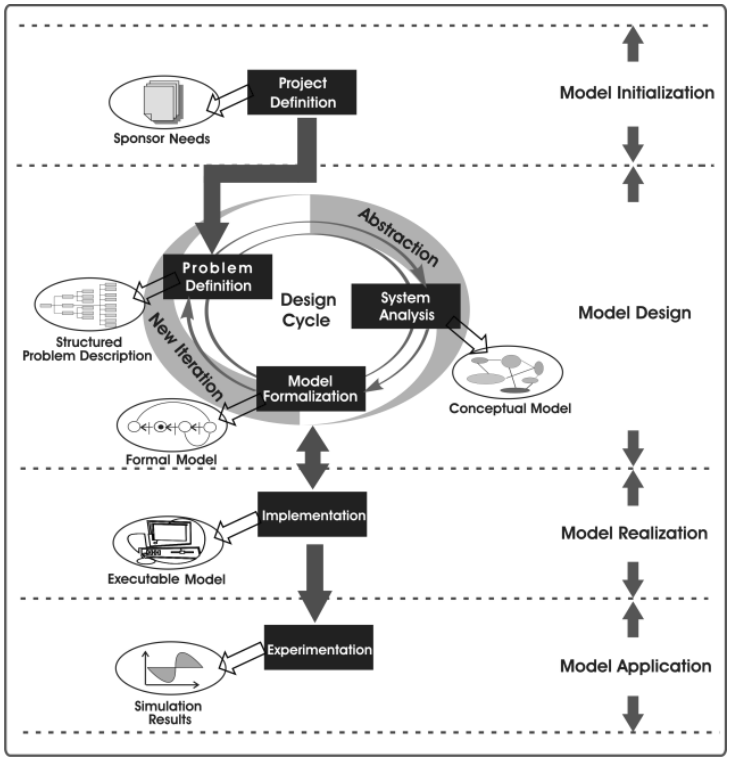

Figure 3. Progress stages of an M\&S project. 
sation, and Simulation Results (SR) are achieved in Model Application.

As a standard process for software and hardware development, the V-Modell XT does not contain all necessary elements specifying the development of simulation models and applications. Therefore, the following missing aspects must be taken into account when applying the V-Modell to conduct an M\&S project:

- Model Design The specification and formalisation of a well-defined simulation model is not a part of software development. During the model design process, the mathematical / logical / graphical / verbal representation of the real system of interest is developed for the objectives of a particular study. Since a typical simulation study requires multifaceted knowledge in different disciplines [15], a variety of representation means such as mathematical equation systems, queuing networks, Petri nets, process algebra [16] and Discrete Event System Specification (DEVS) [17] etc. can be used for developing the work products SPD, CM and FM. The associated activities and roles are also to be integrated in the V-Modell XT. It should be noted that some models, such as the different model types defined in Model Driven Architecture (MDA) [18] proposed by the Object Management Group, could be also established during software development process, however, they (for example models described in UML [19]) serve as aids for specifying the functionalities, the structures, and the behaviors of a software system, and are used in converting a well-formed simulation model into a software and hardware form. This aspect is addressed at the progress stage Model Realisation and completely covered by the V-Modell.

- Data Modelling Throughout the entire M\&S development life cycle, an enormous amount of information must be gathered, analyzed and modeled in terms of qualitative and quantitative data. This task is referred as to data modelling [20]. Three types of data [21] are to be handled: some data are used to specify the model components, and finally, become integrated into the model built; while other data are used either to compare with the simulation results for test purpose or to perform simulation experiments. According to the different applications of input data, data modelling has to closely cooperate with each project progress stage, and therefore, is considered as an integrated part of model development [22]. This aspect has also to be contained in the V-Modell XT-M\&S.

- Model Application Model Application refers to the process of experimenting with the simulation model for a specific purpose, including design of model experiments, execution of simulation runs and interpretation of simulation results. This aspect is out of the consideration range of every software and hardware development process.

\subsection{M\&S Verification and Validation}

As discussed above, the essential M\&S-specific aspects model design, data modelling, and model application are not contained in the V-Modell, while others like model initialisation and model realisation are completely covered. This means that the M\&Sspecific components should be additionally introduced in the V-Modell. For the purpose of model $\mathrm{V} \& \mathrm{~V}$, two issues must be followed:

1. the M\&S-specific elements must be in form and content completely compatible with the VModell XT;

2. appropriate $V \& V$ activities must be defined to evaluate each (intermediate) product of the common V-Modell XT as well as the M\&Sspecific aspects consistently.

To specify the aspects of model design, data modelling and model application in the context of the VModell, the M\&S-specific work products as well as the associated activities and roles have to be additionally defined in the form of process modules. Furthermore, it is also to be determined in which order the new products should be completed and which V\&V activities should be applied to ensure their quality. This means that appropriate project execution strategies and additional decision gates are also to be introduced. More details about the extension of the VModell will be discussed in the next section.

Regarding assessment of product quality, the VModell XT defines concrete requirements for performing V\&V activities. Each product defined in the V-Modell must be evaluated. An evaluation can be conducted either by the developer himself, the socalled self-evaluation, or by independent verification and validation (IV\&V) [12, 23]. In the V-Modell, it is clearly specified whether an IV\&V is required for a product. 


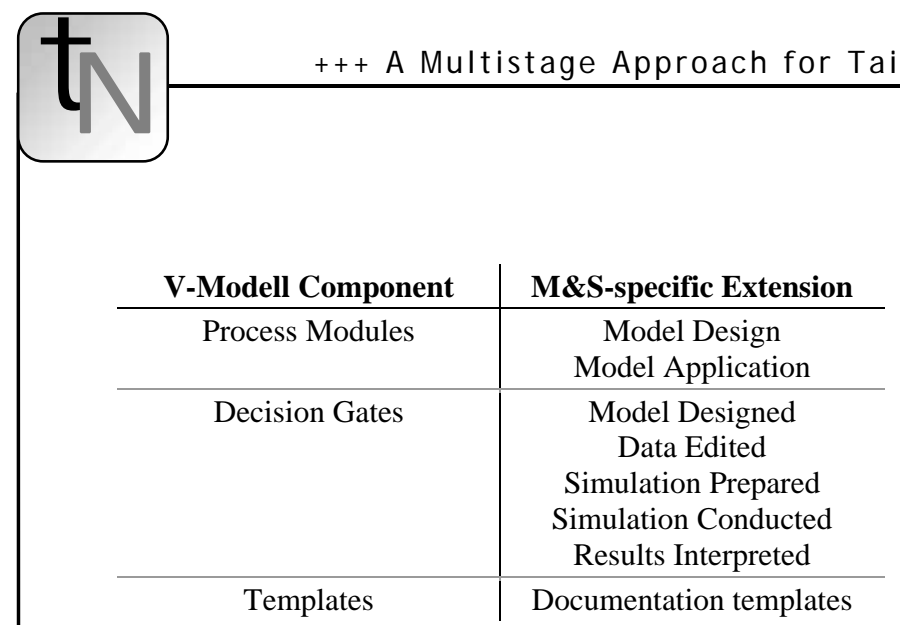

Table 1. M\&S-specific extensions.

The process module Quality Assurance of the VModell is used to specify how and by which means the project quality is intended to be ensured, including the essential $\mathrm{V} \& \mathrm{~V}$ activities for planning, execution and documentation of product evaluation. To achieve unified $\mathrm{V} \& \mathrm{~V}$ of all products, quality assurance measurement must be defined for the M\&Sspecific products consistently with the original products.

Therefore, appropriate $\mathrm{V} \& \mathrm{~V}$ activities should be specified for evaluating each new product involved in Model Design and Model Application by using the same quality assurance mechanism of the V-Modell. Since the quality of data modelling is also a crucial factor for the credibility assessment of M\&S applications, correctness and accuracy of data acquisition, data analysis, data transformation and data use must be estimated in accordance with model $\mathrm{V} \& \mathrm{~V}$ for each model development phase [14, 22]. Thus, evaluation of each individual product and credibility assessment of an overall completed M\&S application can be achieved within the scope of the V-Modell XT.

\subsection{Documentation of Model Development and V\&V}

Documentation is an essential issue for a successful simulation study. However, under pressure, time and cost constraints, model documentation in practical applications is often sacrificed first [24], or conducted only in an arbitrary and informal way $[25,26]$. Suchlike problems of documentation not only reduce the application efficiency of simulation models and make their reuse as well as further development difficult, but also lead to increasing risks of using improper $\mathrm{V} \& \mathrm{~V}$ results. Therefore, a structured and well-defined documentation is required for developing $M \& S$ applications as well as for conducting their $\mathrm{V} \& \mathrm{~V}$.

According to [25], the M\&S documentation should describe detailed information about historical, technical, developmental, maintenance and implementation aspects of a model, including all assumptions, implications and impacts of using the simulation results.
Concerning model verification and validation, not only planning, design and execution of each individual V\&V activity, data used, conclusions, but also separate evaluation results of the intermediate products SPD, CM, FM, EM, and an overall summary should be documented [27]. This documentation approach should be also integrated into the V-Modell $\mathrm{XT}-\mathrm{M} \& \mathrm{~S}$, and corresponds to each phase of model development and V\&V.

\section{Adaptation of Modelling and Simulation Processes}

As described above, an M\&S project requires specific work products, activities, roles and work flows compared to the standard V-Modell XT. This section presents the integration of the M\&S-specific components in compliance with the basic structure of the VModell. Based on the extension, a refined tailoring concept is introduced, which enables the step-by-step adaptation of an $\mathrm{M} \& \mathrm{~S}$ project to suit actual requirements of model development, $\mathrm{V} \& \mathrm{~V}$ and documentation.

\subsection{M\&S-specific Extensions to the V-Modell XT}

Largely in the form of additional process modules and new decision gates, the M\&S-specific components are integrated into the V-Modell as shown in Table 1. Furthermore, useful templates are introduced for the purpose of documentation of model development and V\&V [28]. A more detailed description of the M\&S-specific extensions can be found in $[13,14]$.

The new process module Model Design comprises the necessary activities and work roles to prepare the intermediate products of Structured Problem Description (SPD), Conceptual Model (CM) and Formal Model (FM). Since data modelling cooperates closely with model development, the aspects of the associated data acquisition, data analysis, and data transformation are also specified. Additionally, with respect to model $V \& V$, this process module includes the specification of $\mathrm{V} \& \mathrm{~V}$ requirements for each contained model element, guidelines for definition of test cases and $\mathrm{V} \& \mathrm{~V}$ execution, and documentation of $\mathrm{V} \& \mathrm{~V}$ activities and results.

The other new process module Model Application contains the activities, roles and products required for planning, designing, executing, and documenting model experiments, as well as for interpreting simulation results. The necessary products and activities for the purpose of $\mathrm{V} \& \mathrm{~V}$ are also defined in this process module. 
In order to indicate the milestones in the project sequence, where the M\&S-specific work products have to be evaluated, new decision gates are introduced in project execution strategies for every project type. For an $\mathrm{M} \& \mathrm{~S}$ project of the developer (supplier) side, two additional decision gates Model Designed and Data Edited are defined for ensuring that the M\&Sspecific work products SPD, CM, FM, and the associated input model are completed correctly and accurately. On the other side, for an acquirer (client) project, the new decision gates Simulation Prepared, Simulation Conducted and Results Interpreted enable to conclude that the model experiments are performed as planned, and the observed simulation results are also interpreted appropriately.

To enable a well-defined documentation of model development and $\mathrm{V} \& \mathrm{~V}$, concrete structure and contents requirements for each model element created during the M\&S life cycle are provided and specified in the form of document templates.

\subsection{Tailoring of an M\&S Project in Stages}

In due consideration of the new process modules and decision gates, the M\&S-specific project types including the individual project type variants for an acquirer and a supplier and the corresponding project execution strategies are introduced. Thus, an M\&S project can be principally tailored by using the VModell XT's own tailoring concept, which enables the selection of the mandatory process modules and a suitable project execution strategy in the order of determining the project type, then the possible project type variant, and finally, identifying the values of the

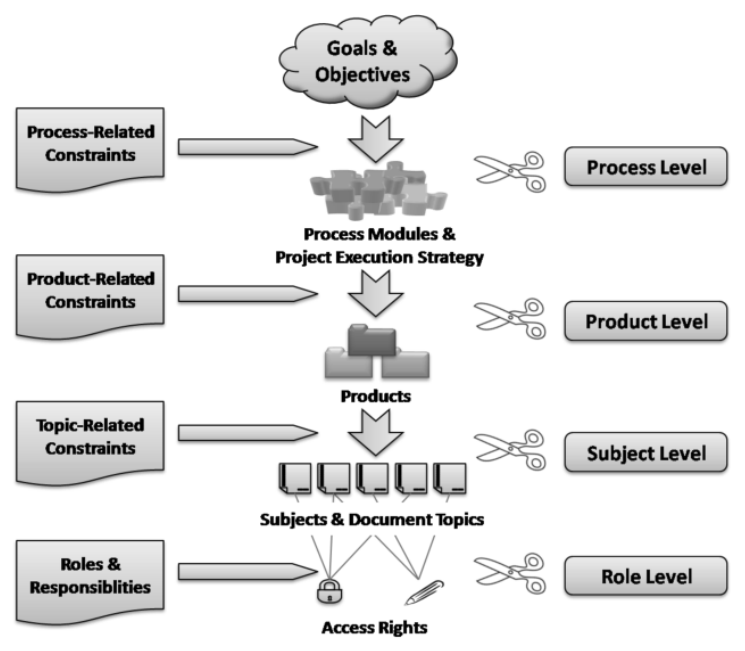

Figure 4. Multistage adaptation of an M\&S project. associated project characteristics. As mentioned previously, there are no additional tailoring steps defined for further adaptation at the product and even at the subject level in the V-Modell XT.

For the purpose of reaching a more precise tailoring decision on a suitable set of necessary work efforts concerning model development, V\&V and documentation in relation to project-specific time, cost and application constraints, a refined adaptation process is defined and applied to conducting an M\&S project. As illustrated in Figure 4, the tailoring efforts of this approach are organized in a hierarchical structure, and the project-specific selection can be made respectively on different levels, viz., the process level, the product level, the subject (or topic) level, and the role level.

Basically, each M\&S-tailoring process starts at the process level. In the event of adaptation at this level, the same tailoring process as in the V-Modell is performed, in which a mandatory set of process modules and a suitable project execution strategy with welldefined decision gates are selected in accordance with identified project requirements and constraints.

Based on more detailed project constraints related to the product level, the already determined process modules can be further tailored. As a result of this process, only relevant work products and their associated $\mathrm{V} \& \mathrm{~V}$ activities for the current project are specified. When further details about how to select essential subjects of a product or to determine concrete documentation topics are available, the subjectrelated adaptation can be arranged.

Compared to the process tailoring, the last two tailoring steps are optional and only applicable, when the corresponding product- and subject-related constraints are identifiable in the course of an M\&S project. Otherwise, all selected process modules must be fully taken into account. Finally, depending on the tailoring results at the process, product, and subject levels, the right to access a particular model element is defined for all involved roles according to their assigned responsibilities.

Similar to the original tailoring concept of the VModell XT, both static and dynamic adaptations are arranged in this tailoring context. Static tailoring refers to selecting required process modules or even products and subjects based on a prescribed and already identified set of requirements and constraints at the beginning of an $M \& S$ project. 
Typically, identifying project requirements and constraints all at once is, however, virtually impossible, for example, the exact impact of using an existing model component or using a particular simulation tool on project execution can be only investigated, when some experiences have been already gained in the context of the current project. In addition, the application profile of a project might be changed during the development process. Therefore, certain modifications of the determined process modules, products, subjects, and the project execution strategy still need to be made in the course of developing an M\&S application. This kind of adaptation is designated as dynamic tailoring.

\section{An Application Example}

This section illustrates how this multistage adaptation process can be used for tailoring a model development project on the supplier side by means of a simple practical example. This example is based on a real simulation project [29], in which a training simulator is to be developed by using some legacy model components available from an early $M \& S$ project. As an extended system component, the completed simulator has to be integrated into an existing computer aided training system environment, and has to cooperate with other components.

In order to keep compatibility with the other existing system components, a specified simulation development tool needs to be applied to the model design and implementation phases of this M\&S project. The hardware part of the simulator comprises the training workstations and other physical devices for networking, operation, display etc. Based on information available at the beginning of the project, tailoring efforts can be arranged within the scope of project planning. As described above, the tailoring process begins at the process level. Namely, the project manager has to determine a mandatory set of process modules and a suitable project execution strategy for the current project.

Like the standard tailoring concept of the V-Modell $\mathrm{XT}$, the M\&S-specific adaptation at the process level is structured as follows:

1. determining the project type,

2. selecting the correct project type variant, and

3. identifying the values of the associated project characteristics.

With respect to the project constraints identified so far, the newly introduced project type "M\&S Devel- opment Project (Supplier)" is to be selected. Process modules to be used for this project type include Drafting and Conclusion of Contract (Supplier), System Development, Delivery and Acceptance (Supplier) and the new process module Model Design in addition to the V-Modell Core, namely Project Management, Quality Assurance, Configuration Management and Problem and Change Management.

Two possible project type variants, namely "Project with Development, Enhancement, or Migration" and "Project with System Maintenance", are available to this project type. Obviously, the former is to be selected in this case. Since the existing model components are applied to the development of this simulator, the predefined project execution strategy "Component-Based Development" is well suited for this project context.

Afterwards, other project characteristics are to be considered. In this example, the project subject characteristic is of major interest. As the training simulator involves software and hardware parts, two more process modules Software Development and Hardware Development are finally identified.

The next step is the tailoring within the selected process modules at the product level. Because no detailed project constraints related to the product level are identifiable at this point in time, all the work products defined in these process modules, especially the M\&S-specific products Structured Problem Description (SPD), Conceptual Model (CM), Formal Model (FM) and Executable Model (EM) are considered as required (or obligatory) in the project plan so far. The same applies to the associated documentation and $\mathrm{V} \& \mathrm{~V}$ activities.

Since the previous tailoring decisions are made according to the project-specific constraints identified at the beginning of the project, these adaptation efforts can be viewed as static tailoring. In addition to static tailoring, further modifications of the already determined work products, the work flows in the project execution strategy, the related documentation and $\mathrm{V} \& \mathrm{~V}$ activities can be made in the course of the model development project. In this case, some adaptations with respect to the development of the work products FM and EM have to be conducted because of the application of the simulation environment "Virtools" [30].

Since the physically correct behaviors and interactions of all the objects considered in the simulation 
study have to be realized by means of the "Havocs Physics Engine" which is integrated in the simulation environment of Virtools, and its associated mechanisms are, however just like a black box, not available to the development team members, it becomes apparent at the time of this M\&S phase that the platform independent Formal Model can not be created and the complete documentation of FM is also impossible according to the project constraints. In the model document of FM, the reasons for this tailoring decision need to be documented.

Similarly, the detailed impact of using this simulation tool on the development of the M\&S product EM can be only identified in the course of the model development. In this project context, the source code of the training simulator is in large part automatically generated by the applied simulation tool. Only the software of the control system and user interface needs to be designed and implemented completely by the development team.

Based on the information obtained at this time, further tailoring decisions relating to the documentation at the subject (or topic) level can be made. Finally, as the result of this adaptation step, the documentation to be conducted for the work product EM includes only:

- description of the overall software and hardware systems involved in the training simulator;

- description of the proprietary development software, namely the control system and user interface.

\section{Conclusion}

Applying a well-structured and flexibly adaptable $M \& S$ process is an essential requirement for effectively developing simulation models and conducting their V\&V. This work introduces a lightweight approach to adaptation of the German standard IT development process, the V-Modell XT, for conducting $\mathrm{M} \& S$ projects. As the V-Modell does not specify some essential M\&S-specific aspects, the related components in terms of model development, V\&V and documentation are additionally defined and integrated in accordance with the basic structure of the VModell, without modifying any existing elements.

In addition, based on the M\&S-specific extensions, a refined tailoring approach is presented, which facilitates the project-specific adaptation of model development, $\mathrm{V} \& \mathrm{~V}$ and documentation at several levels. Furthermore, two different ways of tailoring - static tailoring and dynamic tailoring - are discussed.
While static tailoring reaches a selection decision at the beginning of a project, additional modifications of selected products, activities and documents are subject to dynamic tailoring. The application of this multistage M\&S-tailoring process is then demonstrated by a practical example.

\section{Acknowledgements}

The authors would like to thank the Bundeswehr Transformation Centre (ZTransfBw) and the German Federal Office of Defense Technology and Procurement (BWB) for financial support of the work described in this article.

\section{References}

[1] O. Balci, S. D. Saadi: Proposed Standard Processes for Certification of Modelling and Simulation Applications. In: Proceedings of the 2002 Winter Simulation Conference, 2002.

[2] J. Banks, J. II. Carson, B. Nelson, D. Nicol: DiscreteEvent System Simulation. Forth Edition, Pearson Education International, 2005.

[3] D. Brade, R. Jaquart, J. Voogd, C. Yi: Final State of the REVVA Methodology. In: Proceedings of the 2005 Spring Simulation Interoperability Workshop, San Diego, 2005.

[4] J. Chew, C. Sullivan: Verification, Validation, and Accreditation in the Life Cycle of Models and Simulations. In: Proceedings of the 2000 Winter Simulation Conference, 2000.

[5] S. Hoover, R. Perry: Simulation: a problem-solving approach. Addison-Wesley, 1989.

[6] R. Sargent: Some Approaches and Paradigms for Verifying and Validating Simulation Models. In: Proceedings of the 2001 Winter Simulation Conference, 2001.

[7] V. R. Basili, H. D. Rombach: Tailoring the Software Process to Project Goals and Environments. In: Proceedings of the 9th international conference on Software Engineering, IEEE 1987.

[8] O. Pedreira, M, Piattini, M. R. Luaces, N. R. Brisaboa: A Systematic Review of Software Process Tailoring. ACM SIGSOFT Software Engineering Notes, 32 (3), 2007, pp. 1-6.

[9] M. Broy, A. Rausch: Das neue V-Modell XT - Ein anpassbares Modell für Software und System Engineering. Informatik Spektrum (in German), 28 (3), Springer, 2005, pp. 220-229.

[10] M. Kuhrmann, D. Niebuhr, A. Rausch: Application of the V-Modell XT - Report from a Pilot Project. In: M. Li, B. Boehm, L. Osterweil (eds.): Unifying the Software Process Spectrum, Int. Software Process Workshop, Springer Berlin/Heidelberg, 2005, pp. 463-473. 
[11] A. Rausch, C. Bartelt, T. Ternité, M. Kuhrmann: The V-Modell XT Applied - Model-Driven and DocumentCentric Development. In $3^{\text {rd }}$ World Congress for Software Quality, VOLUME III, Online Supplement, 2005, pp. 131-138.

[12] The V-Modell XT Documentation. Edition 1.3. http://www.v-modell-xt.de, 2009.

[13] Z. Wang: Eine Ergänzung des V-Modell XT zum Einsatz in Projekten der Modellbildung und Simulation. Beiträge der 35. Jahrestagung der Gesellschaft für Informatik (in German), GI-Edition: Lecture Notes in Informatics. Köllen Verlag, Bonn, 2005.

[14] Z. Wang, A. Lehmann: Expanding the V-Modell ${ }^{\circledR} X T$ for Verification and Validation of Modelling and Simulation Applications. In: Proceedings of the $7^{\text {th }}$ International Conference on System Simulation and Scientific Computing, IEEE 2008.

[15] O. Balci: Verification, Validation and Testing. In (J. Bank, eds.) The handbook of Simulation, Chapter 10. John Wiley \& Sons, 1998.

[16] J. Bergstra, J. Klop: Process Algebra for Synchronous Communication. Information and Control, 60 (1/3), 1984, pp. 109-137.

[17] B. P. Zeigler, H. Praehofer, T. Kim: Theory of Modelling and Simulation. Second Edition, Academic Press, 2000.

[18] Object Management Group (OMG): Model Driven Architecture (MDA). http://www.omg.org/mda.

[19] Object Management Group (OMG): Unified Modelling Language (UML). http://www.uml.org.

[20] R. Shannon: Systems Simulation - the Art and Science. Prentice-Hall, 1975.

[21] R. Sargent: Verification, Validation and Accreditation of Simulation Models. In: Proceedings of the 2000 Winter Simulation Conference, 2000.
[22] M. Rabe, S. Spieckermann, S. Wenzel: Verifikation und Validierung für die Simulation in Produktion und Logistik - Vorgehensmodelle und Techniken (in German), Springer-Verlag, Berlin, 2008.

[23] J. D. Arthur, R. E. Nance: Verification and Validation without Independence: A Recipe for Failure. In: Proceedings of the 2000 Winter Simulation Conference, 2000.

[24] O. Balci: Verification, Validation and Accreditation of Simulation Models. In: Proceedings of the 1997 Winter Simulation Conference, 1997.

[25] S. I. Gass: Documenting a Computer-Based Model. Interfaces, 14 (3), 1984, pp. 84-93.

[26] J.P. Kleijnen: Verification and Validation of Simulation Models. European Journal of Operational Research, 82 (1), 1995, pp. 145-162.

[27] R. Sargent: Verification and Validation of Simulation Models. In Proceedings of the 2007 Winter Simulation Conference, 2007.

[28] A. Lehmann, S. Bel Haj Saad, M. Best, A. Köster, S. Pohl, J. Qian, C. Waldner, Z. Wang, Z. Xu: Leitfaden für Modelldokumentation. Abschlussbericht (in German), ITIS e.V., 2005.

[29] Z. Wang, H. Kißner, M. Siems: Applying a Documentation Guideline for Verification and Validation of Simulation Models and Applications: An Industrial Case Study. In: Proceedings of the $7^{\text {th }}$ Industrial Simulation Conference, 2009.

[30] Virtools. http://www.virtools.com, 2008.

Corresponding author: Zhongshi Wang Institut für Technik Intelligenter Systeme (ITIS) Universität der Bundeswehr München D-85577 Neubiberg, Germany zhongshi.wang@unibw.de 